\title{
Hidden Dangers of Biosafety in Schools' Laboratories and the Construction of Management System
}

\author{
Hongbo Zhu* \\ School of Pharmacy, Jiamusi University, Jiamusi 154007, Heilongjiang Province, China \\ *Corresponding author: Hongbo Zhu, zhuhongbo163@163.com
}

\begin{abstract}
With detailed analysis and research on biosafety, it is found that biosafety has an important role and occupies a key position in relation to universities' laboratories. It is also one of the most important components of the safe construction and management of laboratories. At the same time, biosafety and life safety of teachers and students enable orderly and smooth teaching in which they are closely linked. If the biosafety of a university's laboratory has problems, inevitably there would be huge threats to the university itself, teachers, and students. Hence, in order to ensure that laboratories are biologically safe, it is necessary to find out the existing problems, develop flawless solutions and strategies, establish reasonable laboratory safety systems, and strengthen the laboratory protection facilities. Hence, problems pertaining this issue would be properly solved. This article analyzes and studies the construction of a biosafety system in school laboratories for future references.
\end{abstract}

Keywords: School; Laboratory; Biosafety system; Build

Publication date: June 2021; Online publication: June 30, 2021

\section{Introduction}

The reform and opening-up in China have been more than 40 years and the development of the country can be described with the terms; "change" and "by leaps and bounds" especially in view of the current situation in which many schools have set up biological laboratories and expand biological experiment teachings with the constant upgrade of experimental equipment and instruments. In regard to that, there are also many safety hazards in laboratories. In recent years, frequent biosafety accidents have alarmed schools and attracted widespread attention from the society. Hence, at present, one of the most urgent issues that is worth considering is on how to develop a sound and perfect biosafety management system for laboratories. Based on this, this article provides further analysis and research on some safety hazards existing in school laboratories in addition to the construction of a biosafety management system.

\section{Laboratory safety and biosafety}

Teachers and students' personal safety, water and electricity safety, chemical safety, and so on have a close connection and relationship with laboratory safety. One of the most important things is to ensure the safety of personnel. Although biosafety is in the scope of laboratory safety, it is easily neglected. With detailed analysis and research on the understanding of biosafety in laboratories, it involves the engagement of pathogenic microorganism in experiment laboratories and in order to prevent harm by these pathogenic microorganisms on related personnel and the public, there is a need to set up management methods or systems while being equipped with corresponding biological protection measures in the establishment of highly standardized microbiological operation methods and technology integrated strategies. 


\section{Analysis on hidden dangers of biosafety in schools' laboratories}

\subsection{Hidden danger of biological infection}

In the experiment process, there are risks for a few problems including infective events. This would not only have a great impact on the laboratory staffs but would also harm the public health in case of an epidemic breakout which would eventually threaten the stability and harmony of the society. According to relevant investigations in recent years, due to either the lack of a sound and flawless system or inefficient protective measures, the accident rates are high which is a problem existing in all countries.

\subsection{Flawed protection facilities and biosafety management regulations}

In the process of experimental teaching and scientific research in schools' biology laboratories, nearly all operators would prepare specimen pictures, staining microscopy, and other operations which warrant close contact with various pathogenic microorganisms; hence, they are easily affected. At present, biological experiment as the most basic subject in colleges and universities courses requires a freshman or sophomore to conduct them and because these students are neither familiar with these courses or due to low consciousness, problems such as lack of awareness of various specimens in the laboratory may occur. Hence, they often neglect these biological hazards and their impact. Some colleges and universities also direct their students from other non-medical professional courses to be involved in elective experiments including pathogenic biological experiments which may prompt the frequent use of biology laboratories. Hence, in the process of experiment, it increases the risk of hazards. Biology laboratories in some schools still lack professional biosafety facilities in addition to poor biosafety management. The staffs in these laboratories also do not really understand the importance and significance of biosafety management regulations. Therefore, in the actual operation process, problems from illegal operations may arise. Therefore, each school needs to develop a certain laboratory biosafety management system as soon as possible.

\subsection{Flawed pollutants and waste treatment}

In addition, some universities' biosafety investment funds are too little. With the rapid development, there is also a rising trend of prices but the experiment funds in colleges and universities do not increase over time so some schools would repeat experiments using related experiment materials. Moreover, the location where pathogen experiments are held appears messy with discarded materials and laboratory waste disposals are not according to regulations. Private channels are also utilized to purchase and experiment on animals hence, various problems and circumstances would lead to the increase of biosafety hidden dangers.

\section{Methods and strategies for the construction of biosafety system in schools' laboratories}

\subsection{Strengthening the construction of relevant institutions and systems}

"If you want to do a good job, you must first use tools." If schools' laboratories wish to reduce the possibilities and probabilities of biosafety hidden dangers, they need to develop sound and flawless biosafety management systems. Schools need to strengthen their deployment and formulate biosafety rules and regulations for laboratories, ensure that all relevant staffs understand their own responsibilities and obligations, as well as ensure that every single work is carried out and operated in an orderly manner according to relevant procedures. Through the practice of biosafety management in some universities' laboratories, it is proved that the establishment of the biosafety management system as the basis and principle of experiments has a great binding force for the related work. At the school level, there is a need to set up laboratory biosafety management committees with their corresponding roles so that these schools 
are able to conduct comprehensive laboratory safety management work and careful scientific management. The main function involves the distributions and arrangements for daily work such as improving the implementation of the system, form training plans, and so on. At the laboratory level, an individual should be selected to be responsible for safety which includes the general work in laboratories. In addition to that, being proactive and having a positive attitude to constantly improve and perfect the laboratories' infrastructures and equipment are needed among laboratory staffs, teachers, and students in the biosafety training to promote an orderly and smooth work process.

\subsection{Standardizing the disposal of experimental waste and configure emergency supplies}

With detailed analyses and research, it is found that the reason for laboratory biological safety accidents is largely caused by humans, including the experimenter. It is one of the most dangerous factors, hence there is a need to implore all related staffs to adhere to strict management regulations such as laboratory waste management regulations. This includes separating syringes, blades, and other sharps in containers, disinfecting and cleaning Petri dishes containing bacteria including test tubes, reprocessing experimental animals according to schools' requirements, as well as separately storing and labelling harmful substances as hazardous substance. After the completion of experiments, the materials and equipment should be returned to their original places. At the same time, in order to meet the needs of emergency situations, it is necessary to configure laboratory emergency supplies such as protective equipment, disposable gloves, disposable protective clothing, medical masks, medical caps, etc. with adequate preparation of disinfecting supplies such as iodine volt disinfectant, alcohol disinfectant, etc. Other emergency supplies also include band-aids, gauze, cotton swabs, eye washers, etc.

\subsection{Strengthening supervision and management}

If a flawless system is constructed without relevant supervision and administration, the biosafety in laboratories would only be superficial and not effectively implemented. Hence, amidst daily work, laboratories' biological safety management staffs should constantly review in order to conduct careful analyses and comprehensive discussions. In regard to local and foreign biological safety laws and regulations, there is a need to formulate clear rules and regulations by relevant schools which are specifically planned for within the jurisdiction of their laboratories. This includes strict supervisions and inspections of every link in the biological safety check. If in the process of inspection, there are some problems, it is necessary to first, put forward the problem and to eliminate the root of these security hazards. In addition, punitive measures should also be developed for those who violate the laboratory provisions by corresponding punishments to ensure the effect of an orderly system with smooth operations and developments.

Moreover, there is a necessity to increase the investments for laboratories' biosafety. In regard to that, universities should raise investments according to the actual situation of the schools' development, promote the orderly development of related work, and further improve the level and quality of biosafety managements. These investments can be divided into two aspects which are the investments of manpower and of funds. Investments would benefit in the rapid set up of biosafety laboratories which are equipped with relevant safety protection infrastructures and also, in relation to the laboratory waste treatment funds.

\subsection{Enhance students' awareness of life safety}

At present, it is understood that at some students' self-protection awareness is still very poor, hence schools need to focus on this issue. In the experiment process, teachers should conduct regular inspections, remind students that illegal operations should not be conducted, and so on. 


\section{Conclusion}

All in all, a secure and stable operation of laboratories, smooth laboratory teachings and scientific research works, as well as the development of life sciences in China are closely linked with the biosafety of laboratories. Therefore, the related management staffs should have a responsible and serious attitude while implementing laboratory management systems, standardizing experimental operations, as well as regularly carrying out self-inspection with a check list to ensure effective biosafety managements in laboratories and the personal safety of scientific research staffs.

\section{Disclosure statement}

The author declares no conflict of interest.

\section{References}

[1] Yang P, Cui Y, Liu M, 2012, Construction of biosafety management system for animal medical teaching laboratory. Experimental Technology and Management, 29(09): 191-3.

[2] Xia J, Sun P, 2012, Discussion on biosafety management system of university laboratory. Experimental Technology and Management, 29(12): 9-12.

[3] Pan F, Li S, Yang N, et al., 2020, Discussion on biosafety construction of animal-derived microorganism laboratory in universities and research institutes. Basic Medical Education, 22(05): $330-2$.

[4] Zhang X, Wang H, Chen Q, et al., 2020, Construction of biosafety in laboratory of animal medicine teaching in colleges and universities. Education Modernization, 7(29): 152-5,165.

[5] Zhang X, Li C, 2020,. Biochem Technol, 6(04): 110-3.

[6] $\mathrm{Lu} \mathrm{J,} \mathrm{Gu} \mathrm{B,} \mathrm{Xu} \mathrm{H,} \mathrm{2020,} \mathrm{Research} \mathrm{on} \mathrm{biosafety} \mathrm{in} \mathrm{laboratory} \mathrm{of} \mathrm{local} \mathrm{medical} \mathrm{colleges.} \mathrm{Laboratory}$ Research and Exploration, 39(08): 296-9.

[7] Wand Y, Tao Q, Ren H, et al., 2014, Discussion on biosafety management in university laboratory. Laboratory Research and Exploration, 33(09): 301-4. 